\title{
Hints from Run 1 and Prospects from Run 2 at ATLAS
}

\author{
Catrin Bernius on behalf of the ATLAS Collaboration ${ }^{1, a)}$ \\ ${ }^{1}$ New York University (NYU), 4 Washington Pl, New York, NY 10003, United States \\ ${ }^{a}$ Corresponding author: Catrin.Bernius@cern.ch
}

\begin{abstract}
The Large Hadron Collider at CERN has allowed the ATLAS experiment to collect a large amount of proton-proton collision data at $7 \mathrm{TeV}$ and $8 \mathrm{TeV}$ centre-of-mass energies throughout Run 1. This dataset was used to discover a Higgs boson with Standard Model-like properties at a mass of about $125 \mathrm{GeV}$. Furthermore, an impressive number of searches for deviations from the Standard Model expectations have been carried out. To date, no evidence for new physics beyond the SM has been found. However, a few hints in form of 2-3 $\sigma$ deviations have been observed. After an 18-month shutdown, in which the ATLAS detector has undergone various upgrades, the LHC has again started to deliver collision data at an increased centre-of-mass energy of 13 $\mathrm{TeV}$, providing a much improved sensitivity for various searches, in particular for high mass particles. Some representative hints at the LHC Run 1 are presented, a brief overview of ATLAS upgrades and prospects for SUSY searches with early Run 2 data are given.
\end{abstract}

\section{Introduction}

The Standard Model (SM) of particle physics has been extraordinarily successful at describing the fundamental particles which are responsible for forces and matter. The discovery of the Higgs boson in 2012 at the CERN Large Hadron Collider (LHC) [1] by the ATLAS and CMS collaborations [2,3] has proven the success of the SM. The Higgs boson discovery $[4,5]$ has completed the SM, but several phenomena, including dark matter, CP violation and gravity, remain unaccounted for. Furthermore, the presence of the Higgs boson introduces a hierarchy problem where radiative corrections to the Higgs mass must be extremely finely tuned to ensure that there is cancellation with the bare mass. Searches for supersymmetric and exotic phenomena are part of the rich physics program of the LHC that could provide solutions to the many unsolved questions. Brief summaries of results from the Standard Model, top and Higgs sector will be given, followed by some selected results with an emphasis on possible hints in form of 2-3 $\sigma$ deviations. Then a brief summary of the upgraded ATLAS detector together with an outlook to supersymmetry (SUSY) prospects in Run 2 is given.

\section{The ATLAS Detector}

The ATLAS detector is one of two multi-purpose detectors with a cylindrical geometry at the Large Hadron Collider (LHC). It comprises an inner detector (ID) surrounded by a thin superconducting solenoid, a calorimeter system and an extensive muon spectrometer in a toroidal magnetic field. The ID tracking system consists of a silicon pixel detector, a silicon micro-strip detector (SCT), and a transition radiation tracker (TRT). It provides precise position and momentum measurements for charged particles and allows efficient identification of jets containing b-hadrons (b-jets) in the pseudorapidity range $|\eta|<2.5$. The ID is immersed in a $2 \mathrm{~T}$ axial magnetic field and is surrounded by highgranularity lead/liquid-argon (LAr) sampling electromagnetic calorimeters which cover the pseudorapidity range $|\eta|<$ 3.2. A steel/scintillator tile calorimeter provides hadronic energy measurements in the central pseudorapidity range $(|\eta|<1.7)$. In the forward regions $(1.5<|\eta|<4.9)$, the system is complemented by two end-cap calorimeters using LAr as active material and copper or tungsten as absorbers. The muon spectrometer (MS) surrounds the calorimeters and consists of three large superconducting eight-coil toroids, a system of tracking chambers, and detectors for triggering. The deflection of muons is measured within $|\eta|<2.7$ by three layers of precision drift tubes, and cathode strip chambers in the innermost layer for $|\eta|>2.0$. The trigger chambers consist of resistive plate chambers in the barrel 
$(|\eta|<1.05)$ and thin-gap chambers in the end-cap regions $(1.05<|\eta|<2.4)$. A three-level trigger system is used to select events. A hardware-based Level-1 trigger uses a subset of detector information to reduce the event rate to a value to $75 \mathrm{kHz}$ or less. The rate of accepted events is then reduced to about $400 \mathrm{~Hz}$ by two software-based trigger levels, Level-2 and the Event Filter.

\section{Hints from Run 1}

This section will give an overview of selected results for Standard Model, Top and Higgs physics and searches for SUSY and new physics.

\section{Standard Model}

SM processes have been measured with high precision at the LHC in proton-proton collisions. Figure 1 shows a summary of ATLAS SM cross section measurements [6], and demonstrate the broad range of SM measurements that have been carried out. A good agreement with SM expectations within the uncertainties can be seen. The experimental uncertainties are in some cases at the level of the theoretical predictions, in some even better.

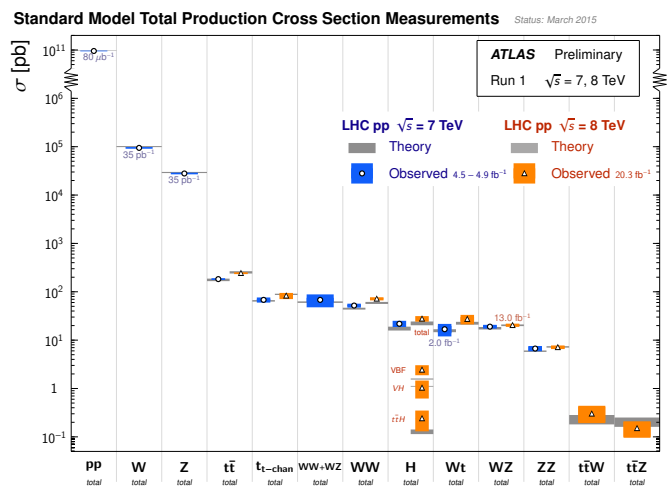

FIGURE 1: Summary of several Standard Model total production cross section measurements [6], corrected for leptonic branching fractions, compared to the corresponding theoretical expectations. All theoretical expectations were calculated at NLO or higher.

\section{Top Production and Mass Measurement}

At the LHC, top quarks are predominantly produced in pairs via the strong interaction, contributing by a factor of about $80 \%$ to the total top quark pair production cross section. The calculation of the top quark pair cross section has recently been completed to exact next-to-next-to leading order (NNLO) in QCD including resummation of nextto-next-to-leading logarithmic (NNLL) soft gluon terms. Figure 2 shows the summary of the top-pair production cross-section measurements of the ATLAS experiment [7].

The latest precision measurement of the top quark mass in the $t \bar{t} \rightarrow$ lepton + jets and $t \bar{t} \rightarrow$ dilepton channels using a 3D fit to reduce effect of Jet Energy Scale (JES) and bJES on the final measurement [8]. Figure 3 shows the Likelihood contours with the correlation determined in data of the measured $m_{\text {top }} \rightarrow$ lepton+jets to the JSF (left) and the bJSF (right). The combination of lepton + jets and dilepton channels gives a measured top mass of $m_{\text {top }}=172.99 \pm 0.48($ stat $) \pm 0.78($ syst $) \mathrm{GeV}$ with a total uncertainty of $0.91 \mathrm{GeV}$.

\section{Higgs: The Search that Became a Measurement}

Since the discovery of the Higgs boson [4, 5], an entire new field has emerged. The observed Higgs boson is to a good precision compatible with the SM Higgs boson due to the following observations:

- $\quad$ direct evidence of coupling to $\mathrm{W}$ and $\mathrm{Z}$, 


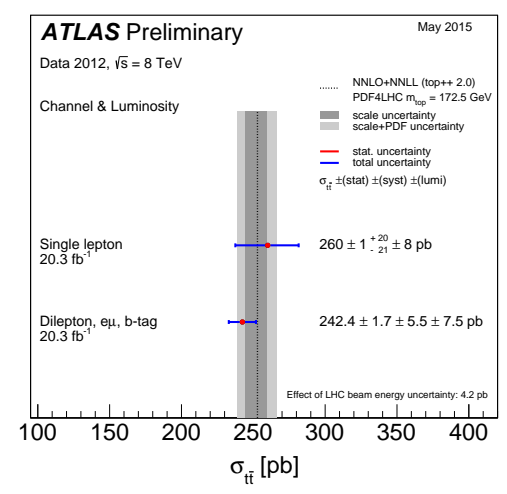

FIGURE 2: Summary of measurements of the top-pair production cross-section on ATLAS at $8 \mathrm{TeV}$ compared to the exact NNLO QCD calculation complemented with NNLL resummation (top++2.0 ) [7]. The theory band represents uncertainties due to renormalisation and factorisation scale, parton density functions and the strong coupling. The measurements and the theory calculation is quoted at $m_{\text {top }}=172.5 \mathrm{GeV}$.
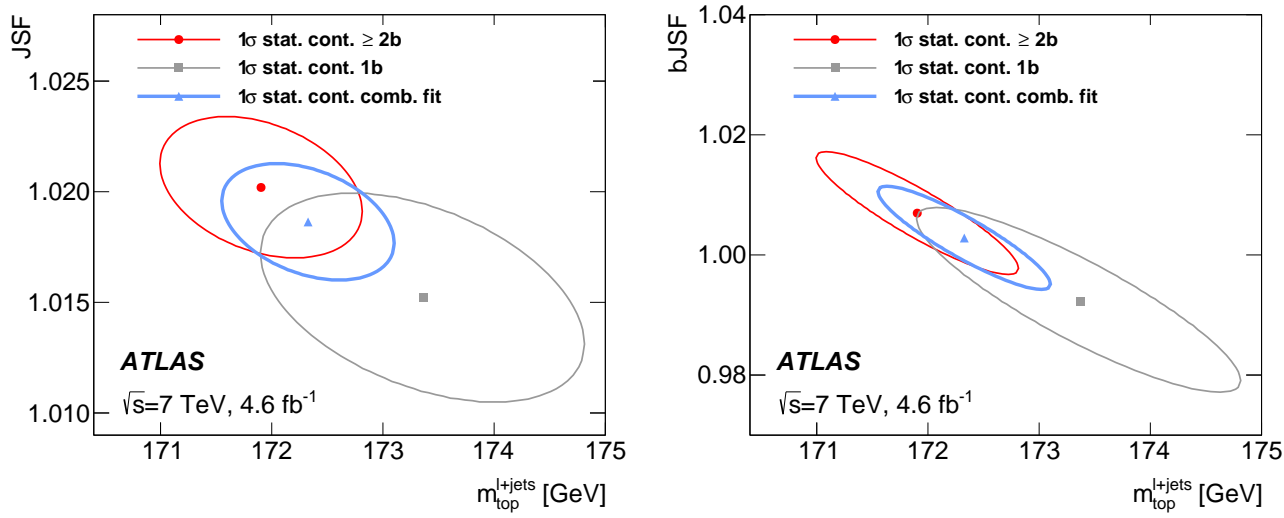

FIGURE 3: Likelihood contours showing the correlation determined in data of the measured $m_{\text {top }}$ in the lepton+jets analysis to (a) the JSF (left) and the bJSF (right) within the $t \bar{t} \rightarrow$ lepton+jets analysis [8].

- $\quad$ direct evidence of coupling to taus (and therefore to fermions),

- direct evidence for non-universal couplings,

- evidence for VBF production,

- indirect evidence of coupling to top quarks,

- evidence of the scalar nature.

Figure 4 shows the updated measurements of the signal-strength parameter from a simultaneous fit to all decay channels that were analysed. The measurements are consistent and compatible with a single value with a probability of $76 \%$. Assuming a common multiplier to all signal yields, they can be combined to result in a global signal-strength value which is consistent with the SM expectation of $=1$ with a probability of $18 \%$ of [9].

Establishing the properties of the Higgs boson has already been extensively studied during Run 1 and will continue to be studied through collaboration with the theory community and with the data from Run 2. Two new key SM Higgs measurements have submitted since November 2014: The $6.1 \sigma$ (5.8 exp) observation of the Higgs boson in the channel $H \rightarrow W W^{*}[10]$ and the $4.5 \sigma$ observation in the channel $H \rightarrow \tau \tau$ [11].

\section{Search for Supersymmetric Particles}

The dominant production mode for SUSY particles at the LHC is through strong production of squark-gluino, squarksquark and gluino-gluino pairs which then decay in cascades to Lightest SUSY Particles (LSP). There are also quite 


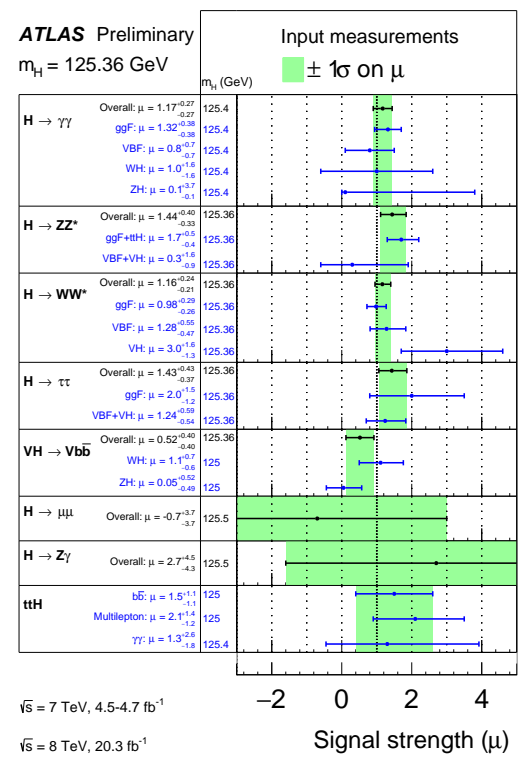

FIGURE 4: The observed signal strengths and uncertainties for different Higgs boson decay channels and their combination for $m_{H}=125.36 \mathrm{GeV}$. Higgs boson signals corresponding to the same decay channel are combined together for all analyses. The best-fit values are shown by the solid vertical lines. The total $\pm \sigma$ uncertainties are indicated by green shaded bands, with the individual contributions from the statistical uncertainty (top), the total (experimental and theoretical) systematic uncertainty (middle), and the theory systematic uncertainty (bottom) on the signal strength shown as horizontal error bars [9].

strong motivations to study SUSY at the electroweak scale, e.g. the hierarchy problem, gauge group unification, etc. Figure 5 shows a summary of ATLAS searches for electroweak production of charginos and neutralinos [12].

An analysis with a deviation from the background-only hypothesis is the SUSY search with same-flavour lepton pair with opposite charge, jets and large missing transverse momentum in the final state at $\sqrt{s}=8 \mathrm{TeV}$ and an integrated luminosity of $20.3 \mathrm{fb}^{-1}$ [13]. The two decay channels, in which this final state can be observed are the:

- decays of squarks and gluinos with $Z$ bosons in the final state $(Z \rightarrow l l)$ resulting in a peak in the di-lepton invariant mass distribution $\left(m_{l l}\right)$ around the $Z$-boson;

- decays of heavy neutralinos leads to a rising distribution in mll that terminates at a kinematic endpoint (events with larger $m_{l l}$ values would violate energy conservation in the decay of a neutralino).

In the squark/gluino case, an excess of events above the expected SM background with a significance of $3 \sigma(1.7 \sigma)$ in the $e e(\mu \mu)$ channel has been observed, in the neutralino case, the data is well-described by expected SM background.

\section{Hints from New Physics Searches}

Various hints in form of 2-3 $\sigma$ deviations have been observed in the searches for new physics, but only a few selected analyses will be very briefly described here.

Di-boson resonances are predicted in several extensions to the SM, such as technicolor, warped extra dimensions and Grand Unified Theories (GUTs). In the high mass di-boson resonance searches with boson-tagged jets at $\sqrt{s}$ $=8 \mathrm{TeV}$ [14], $W$ and $Z$ bosons are produced together with large transverse momentum relative to their mass. Each boson is reconstructed in a single large-radius jet. The resonance structure is then looked for on a smoothly falling di-jet invariant mass spectrum. The most significant discrepancy with the background-only model occurs around 2 $\mathrm{TeV}$ in the $W Z$ channel with a local significance of $3.4 \sigma$. A global significance with the entire mass range in all three channels $(W Z, Z Z, W W)$ of $2.5 \sigma$ has been found. Figure 6a shows the background-only fit to the di-jet mass distribution in data for $W Z$ selection

Several Beyond the Standard Model (BSM) processes predict final states with $b$-jets, leptons with the same charge and missing transverse momentum $E_{\mathrm{T}}^{\text {miss }}[16]$, while the production via SM processes is rare. These models 


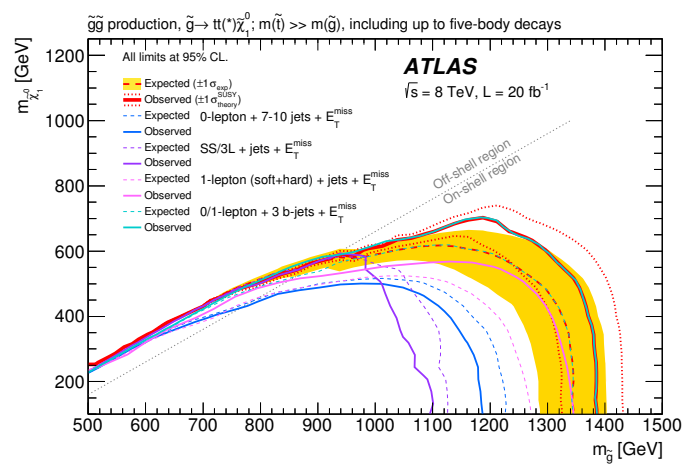

FIGURE 5: Summary of ATLAS searches for electroweak production of charginos and neutralinos based on $20 \mathrm{fb}^{-1}$ of pp collision data at $\sqrt{(s)}=8 \mathrm{TeV}$. Exclusion limits at $95 \%$ confidence level are shown in the chargino and neutralino mass plane. The dashed and solid lines show the expected and observed limits, respectively, including all uncertainties except the theoretical signal cross section uncertainties [12].

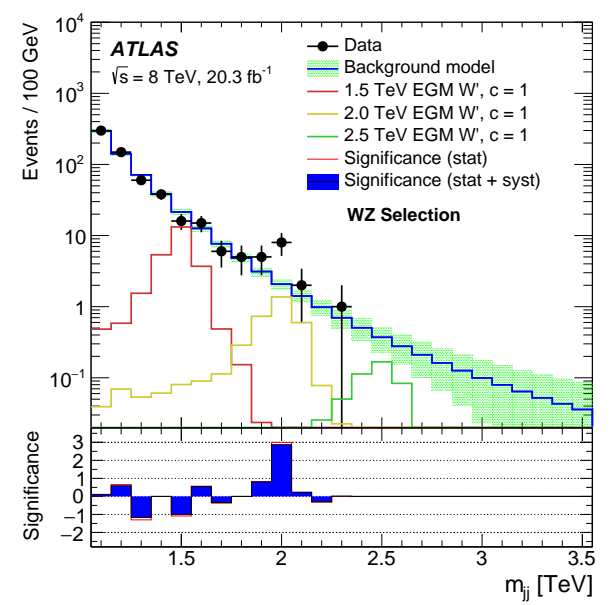

(a) Background-only fit to the di-jet mass $\left(m_{j j}\right)$ distribution in data for the $W Z$ selection [14]. The significance in the inset is the difference between the data and the fit in units of the uncertainty on this difference.

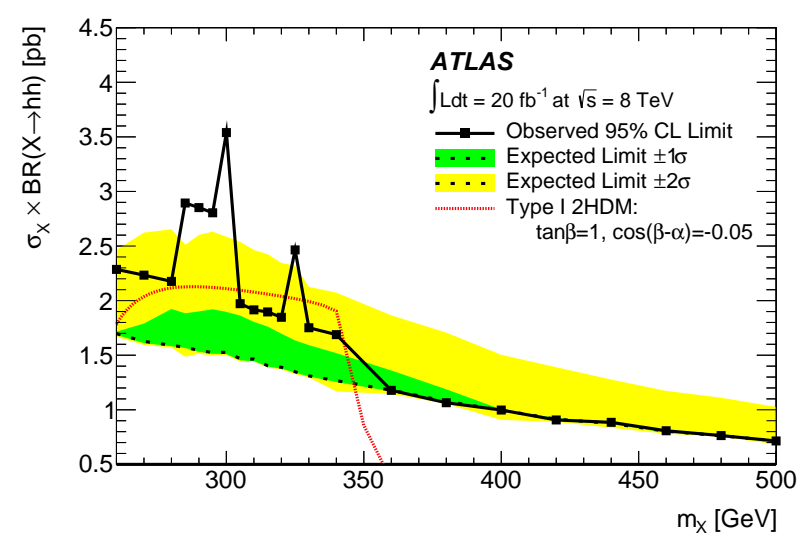

(b) $95 \%$ CL upper limit on the cross section times branching ratio of a narrow resonance decaying to pairs of Higgs bosons as a function of $m_{X}[15]$.

FIGURE 6: Hints from searches for new physics.

include vector-like quarks, chiral $b^{\prime}$-quark pair production, two positively charged top quarks and the 4-top production. The search performed considers all these models by defining eleven signal regions, the subsets of these regions are combined when searching for each class of models. In the three signal regions primarily sensitive to positively charged top quark pair production, the data yield is consistent with the background expectation. More data events than expected from background are observed in the set of eight signal regions defined for the vector-like quarks and chiral $b^{\prime}$-quarks searches, however the significance of the discrepancy is less than $2 \sigma$. The discrepancy reaches $2.5 \sigma$ in the set of five signal regions defined for searching for four-top-quark production.

Searches for non-SM physics can be carried out with events consistent with either resonant $(X \rightarrow h h)$ or nonresonant pair production of Higgs bosons. Looking at the clean final state $h h \rightarrow b b \gamma \gamma$, a small excess of $2.1 \sigma$ was found at $m_{X}$ of around $300 \mathrm{GeV}$ [15]. Figure $6 \mathrm{~b}$ shows the $95 \% \mathrm{CL}$ upper limit on the cross section times branching ratio of a narrow resonance as a function of $m_{X}$. 


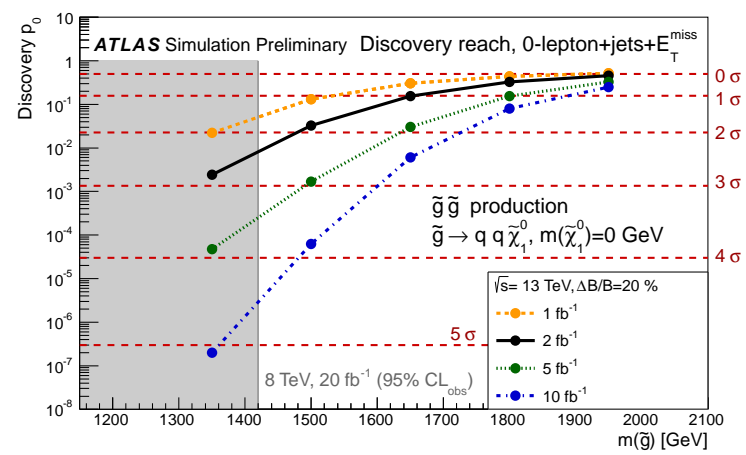

(a) Gluino-pair production with a 0-lepton + jets $+E_{\mathrm{T}}^{\mathrm{miss}}$ final state.

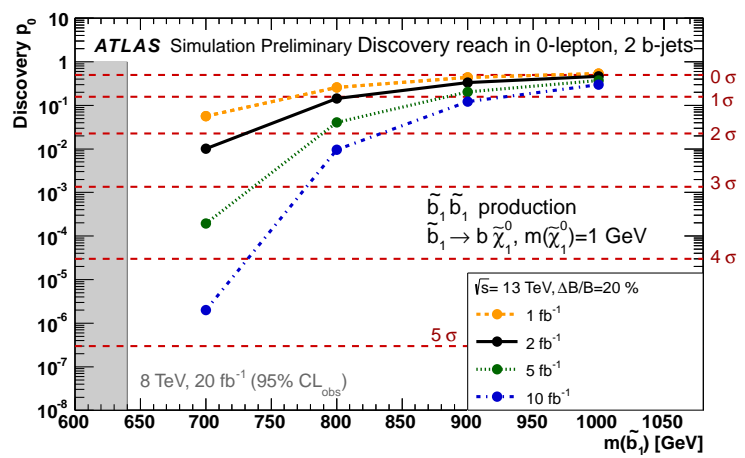

(b) Sbottom-pair production with a 0-lepton +2 b-jets final state.

FIGURE 7: The p-values for a SM background only hypothesis for the total integrated luminosity as a function of the gluino and sbottom masses, each plot corresponding to a fixed uncertainly of 20 or $25 \%$.

\section{Prospects for Run 2}

During the long shutdown period 2013-2015, the ATLAS detector has undergone various upgrades [17]. These include completely new additions like the Insertable B-Layer (IBL), an additional silicon layer for improvement of the tracking performance [18] and additional chambers for complete muon coverage as well as various upgrades of other subdetectors, e.g. the Diamond Beam Monitor, the Beam Condition Monitors, etc. The trigger and data acquisition system also has undergone major upgrades [19], including amongst many others an increased data readout rate of $1 \mathrm{kHz}$ compared to $400 \mathrm{~Hz}$ previously.

While there are many prospects for the discovery of new physics, only the expected sensitivity studies for gluino and squark searches using the early LHC $13 \mathrm{TeV}$ data will be mentioned here. Three cases can be considered, the gluino pair production in final states with large missing transverse momentum and no leptons or exactly one isolated lepton and the bottom squark pair production [20]. The p-values for a SM background only hypothesis are shown for two of the three cases in Figure 7.

\section{REFERENCES}

[1] L. R. Evans and P. Bryant, JINST 3 S08001 (2008).

[2] ATLAS Collaboration, JINST 3 S08003 (2008).

[3] CMS Collaboration, JINST 3 S08004 (2008).

[4] ATLAS Collaboration, Phys. Lett. B 716, 1-29 (2012).

[5] CMS Collaboration, Phys. Lett. B 716, p. 30 (2012).

[6] https://atlas.web.cern.ch/Atlas/GROUPS/PHYSICS/CombinedSummaryPlots/SM.

[7] https://atlas.web.cern.ch/Atlas/GROUPS/PHYSICS/CombinedSummaryPlots/TOP .

[8] ATLAS Collaboration, Eur. Phys. J. C 75, p. 330 (2015).

[9] ATLAS Collaboration, ATLAS-CONF-2015-007 .

[10] ATLAS Collaboration, Phys. Rev. D 92 (2015).

[11] ATLAS Collaboration, JHEP 04, p. 117 (2015).

[12] https://atlas.web.cern.ch/Atlas/GROUPS/PHYSICS/CombinedSummaryPlots/SUSY .

[13] ATLAS Collaboration, Eur. Phys. J. C 75, p. 318 (2015).

[14] ATLAS Collaboration, (2015), arXiv:1506.00962 [hep-ex] .

[15] ATLAS Collaboration, Phys. Rev. Lett. 114, p. 081802 (2015).

[16] ATLAS Collaboration, (2015), arXiv:1504.04605 [hep-ex] .

[17] ATLAS Collaboration, Tech. Rep. CERN-LHCC-2011-012. LHCC-I-020 (CERN, Geneva, 2011).

[18] W. Lukas, Tech. Rep. ATL-INDET-PROC-2014-008 (CERN, Geneva, 2014).

[19] ATLAS Collaboration, Tech. Rep. CERN-LHCC-2013-018. ATLAS-TDR-023 (CERN, Geneva, 2013).

[20] ATLAS Collaboration, Tech. Rep. ATL-PHYS-PUB-2015-005 (CERN, Geneva, 2015). 